\title{
Coil Occlusion of Patent Ductus Arteriosus - Impact of 0.052-Inch Gianturco Coil Without Amplatzer Duct Occluder
}

\author{
Hideshi Tomita, MD; Motoki Takamuro, MD; Shigeto Fuse, MD; Norihisa Horita, MD; \\ Kinya Hatakeyama, MD; Hiroyuki Tsutsumi, MD; Satoshi Yazaki, MD*; \\ Shigeyuki Echigo, MD*; Kohji Kimura, MD**
}

\begin{abstract}
Background Coils are the only devices available for transcatheter occlusion of patent ductus arteriosus (PDA) in Japan. Since April 1999, we have introduced a 0.052-inch Gianturco coil (0.052-inch coil) to close PDA $\geq 2.5 \mathrm{~mm}$. Methods and Results A retrospective survey of the outcome of coil occlusions for PDA $\geq 2.5 \mathrm{~mm}$ before and after the 0.052 -inch coil became available found that (1) the frequency of PDA $\geq 2.5 \mathrm{~mm}$ among all candidates for coil occlusion significantly increased after the availability of a 0.052 -inch coil $(\mathrm{p}<0.01)$; (2) deployment complicated by migration $(\mathrm{p}<0.01)$, and prolonged procedure time $(\mathrm{p}<0.05)$ were significantly decreased after the introduction of the 0.052 -inch coil. In a multivariate logistic regression model for uneventful deployment adjusted for age, pulmonary to systemic flow ratio, and use of a 0.052-inch coil, use of the 0.052-inch coil significantly decreased eventful deployment ( $\mathrm{p}<0.05$ ); and (3) successful deployment of a coil for PDA $\geq 4 \mathrm{~mm}$ significantly increased with the 0.052 -inch coil $(\mathrm{p}<0.01)$. Complete occlusion was achieved once deployment was successful. Conclusion Introduction of the 0.052-inch coil decreased complicated coil occlusion deployment for PDA $\geq 2.5 \mathrm{~mm}$, and contributed to a better likelihood of coil occlusion for PDA $\geq 4 \mathrm{~mm}$. (Circ J 2006; 70: 28-30)
\end{abstract}

Key Words: Coil occlusion; Flipper coil; 0.052-inch Gianturco coil; Patent ductus arteriosus

$\mathbf{O}$ clusion of a patent ductus arteriosus (PDA) with a coil has become the common treatment strategy in Japan since the detachable PDA coil (Flipper coil $^{\circledR}$, Cook Inc, Bloomington, IN, USA) was released!-4 Although, the Amplatzer duct occluder, which can effectively close medium- to large-sized PDAs, is currently available in many countries, it is not yet approved in Japan. The Porstmann method has been reported as useful in adults, but has been almost abandoned for children because of the large applicator required to insert the Ivalon plug from a femoral artery. Other devices for transcatheter closure of PDA, such as the Rashkind PDA occluder ${ }^{7}$ or the Grifka bag, are also not approved. Consequently, in Japan, the coil is the only device currently available for transcatheter closure of PDA in children.

Grifka et al first reported using a 0.052-inch Gianturco coil (0.052-inch coil, Cook Inc) with controlled delivery using a biotome? As our current policy is to use a 0.052 inch coil for PDA $\geq 2.5 \mathrm{~mm}$, we retrospectively analyzed the impact of using this coil in the setting of it being the only device available for transcatheter closure of PDA.

(Received July 19, 2005; revised manuscript received October 11, 2005; accepted November 4, 2005)

Department of Pediatrics, Sapporo Medical University School of Medicine, Sapporo, Departments of *Pediatrics and **Radiology, National Cardiovascular Center, Suita, Japan

Mailing address: Hideshi Tomita, MD, PhD, Department of Cardiology, Hokkaido Children's Hospital and Medical Center, 1-10-1 Zenibako, Otaru, Hokkaido 047-0261, Japan. E-mail: tomitah@ sapmed.ac.jp

\section{Methods}

From March 1995 to December 2004, a single operator (HT) performed 160 transcatheter occlusions in 145 patients with PDA at the Sapporo Medical University Hospital and National Cardiovascular Center. Of these, outcomes and complications were retrospectively analyzed from the medical records of 61 coil occlusions in 59 patients with a native PDA $\geq 2.5 \mathrm{~mm}$. The angiographic appearance of the PDA was classified according to Krichenko et al ${ }^{10}$ and the minimum diameter of the PDA was measured on the lateral projection of the aortogram. All patients had follow-up intervals longer than 6 months. Cases of coil occlusions for residual shunt following surgery or a previous transcatheter occlusion were excluded.

From March 1995 to March 1999, we used the 0.038-inch Gianturco coil (Cook Inc) or Flipper coil deployed either retrogradely or progradely. A snare catheter was occasionally used with the Gianturco coil for control of delivery as reported by Sommer et al!1 Since April 1999, we have used the 0.052 -inch Gianturco coil for PDA $\geq 2.5 \mathrm{~mm}$, the procedure for which is reported elsewhere,,2,13 Briefly, 3F "cup" biopsy forceps are advanced through a $4 \mathrm{~F}$ sheath. The round-tipped leading end of the 0.052 -inch coil is extruded beyond its metal housing and pulled slightly by mosquito forceps. The extended tip is then carefully grasped with the biopsy forceps and after tightening the forceps, the coil is gently retracted into the sheath. We routinely use a $6 \mathrm{~F}$ Brite tip guiding catheter (Johnson and Johnson) as the delivery catheter, which is passed across the PDA either progradely or retrogradely. The 4F sheath, loaded with the coil-biotome, is inserted into the hemostatsis valve of the Brite tip, and the coil-biotome is advanced slowly through the Brite tip. 


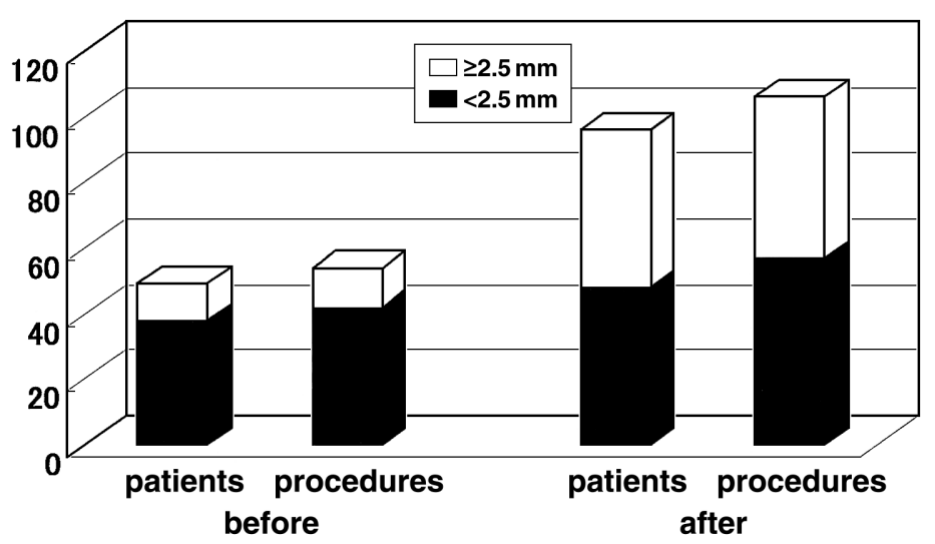

Fig 1. Number of coil occlusions before and after introduction of the Gianturco 0.052-inch coil $(\mathrm{p}<0.01)$.
Table 1 Occlusion of PDA $\geq 2.5 \mathrm{~mm}$ Using the 0.052-Inch Coil

\begin{tabular}{lccc}
\hline \hline & Before coil & After coil & $p$ value \\
\hline No. of patients/procedures & $11 / 12$ & $48 / 49$ & \\
Av. Age (years) & 2.3 & 13.7 & $<0.01$ \\
Qp/Qs & 2.2 & 1.7 & $<0.05$ \\
Diameter (mm) & 3.4 & 3.5 & $N S$ \\
Successful deployment & $8 / 11$ & $45 / 48$ & $N S$ \\
Complete occlusion & $8 / 11$ & $43 / 48$ & $N S$ \\
Procedure time (min) & 74 & 44 & $<0.05$ \\
No. of coils & 3 & 2 & $N S$ \\
Migration & $7 / 12$ & $6 / 49$ & $<0.01$ \\
\hline
\end{tabular}

$P D A$, patent ductus arteriosus; $Q p / Q s$, pulmonary to systemic flow ratio; NS, not significant.

Table 2 Multivariate Logistic Regression Model of Successful Deployment, Adjusted for Age, Qp/Qs, and Use of the 0.052Inch Coil

\begin{tabular}{lccc}
\hline \hline & Odds ratio & \multicolumn{1}{c}{$95 \% \mathrm{CI}$} & p value \\
\hline Age & 1.19 & $0.83-1.72$ & $\mathrm{NS}$ \\
Qp/Qs & 0.42 & $0.12-1.50$ & $\mathrm{NS}$ \\
Use of 0.052-inch coil & 1.77 & $0.23-13.90$ & $\mathrm{NS}$ \\
\hline
\end{tabular}

CI, confidence interval; other abbreviations are in Table 1.

Initially, we delivered the 0.052 -inch coil retrogradely, but currently we use a prograde approach, as controlling the number of loops in the pulmonary artery is easier with a prograde rather than a retrograde approach. Two or 3 coils are deployed simultaneously for a PDA $\geq 3.5-4.0 \mathrm{~mm}$.

We do not have data on fluoroscopic times; however, we analyzed the total procedure time needed to complete deployment of coils.

Statistical analysis was done by chi-square test or multiple logistic regression and a p-value $<0.05$ was regarded as statistically significant.

\section{Results}

\section{Frequency of Coil Occlusion for PDA $\geq 2.5 \mathrm{~mm}$}

Before the availability of the 0.052 -inch coil (pre 0.052inch coil), 54 procedures were performed in 49 patients, whereas after the introduction of the 0.052 -inch coil (post 0.052 -inch coil), there were 106 procedures in 96 patients. Pre 0.052-inch coil, 12 procedures in 11 patients were for a PDA $\geq 2.5 \mathrm{~mm}$, whereas post 0.052 -inch coil, there were 49 procedures in 48 patients. The frequency of PDA $\geq 2.5 \mathrm{~mm}$ among all candidates significantly increased post 0.052 inch coil (Fig 1, p<0.01).
Table 3 Multivariate Logistic Regression Model of Uneventful Deployment, Adjusted for Age, Qp/Qs, and Use of the 0.052Inch Coil

\begin{tabular}{lccc}
\hline \hline & Odds ratio & $95 \%$ CI & p value \\
\hline Age & 1.00 & $0.95-1.05$ & $N S$ \\
Qp/Qs & 0.51 & $0.19-1.39$ & $N S$ \\
Use of 0.052-inch coil & 6.33 & $1.27-31.60$ & $<0.05$ \\
\hline
\end{tabular}

Abbreviations as in Tables 1 and 2.

Table 4 Occlusion of PDA $\geq 4.0 \mathrm{~mm}$ Using the 0.052-Inch Coil

\begin{tabular}{lccc}
\hline \hline & Before coil & After coil & p value \\
\hline Maximal diameter with & 3.8 & 5.6 & - \\
$\quad$ successful deployment $(\mathrm{mm})$ & & & \\
Successful deployment & $0 / 2$ & $10 / 11$ & $<0.01$ \\
Complete occlusion & $0 / 2$ & $10 / 11$ & $<0.01$ \\
\hline
\end{tabular}

Abbreviation as in Table 1.

\section{Underlying Patient Characteristics}

Age and body weight post 0.052 -inch coil were greater than pre 0.052 -inch coil, because there were 4 adult patients post 0.052 -inch coil. However, there were no significant differences between the 2 time periods in the minimum diameter of PDA, distribution of angiographic morphology. Pulmonary to systemic flow ratio (Qp/Qs) was slightly larger in pre 0.052 -inch coil than in post 0.052-inch coil (Table 1).

\section{Outcome of Coil Occlusions Pre and Post 0.052-Inch Coil for $P D A \geq 2.5 \mathrm{~mm}$}

There were no significant differences between the 2 periods in the ratio of successful deployment and complete occlusion, or in the number of coils deployed. "Eventful" deployments complicated by migration $(\mathrm{p}<0.01)$, or long procedure time $(\mathrm{p}<0.05)$ were significantly decreased post 0.052 -inch coil (Table 1 ). Multivariate analysis using a regression model of successful deployment adjusted for age, $\mathrm{Qp} / \mathrm{Qs}$, and use of the 0.052-inch coil showed no significant contribution of use of the 0.052-inch coil to successful deployment (Table 2).

However, the multivariate analysis of uneventful deployment showed a significant contribution of the use of the 0.052-inch coil for uneventful deployment (Table 3).

Hemolysis was a complication in 3 adult patients (before, 1 ; after, 2).

Coil Occlusion for PDA $\geq 4.0 \mathrm{~mm}$

The maximum PDA diameter for which there was suc- 
cessful deployment in each period was $3.8 \mathrm{~mm}$ and $5.6 \mathrm{~mm}$, respectively, and successful deployment of the coil for PDA $\geq 4 \mathrm{~mm}$ significantly increased post 0.052 -inch coil $(p<0.01)$. Complete occlusion was achieved once deployment was successful (Table 4).

\section{Discussion}

Transcatheter occlusion with Gianturco coils is a safe and effective method of occluding a PDA, $1,2,8$ but there have been reports of complications including migration of the coil, hemolysis, and recanalization, particularly when the minimum diameter of the ductus exceeds approximately $3.0 \mathrm{~mm}^{4,14}$ The modified Grifka method ${ }^{9}$ using the 0.052 inch coil offers better positioning during implantation, because the coil keeps its tightly wound loop size and configuration, and consequently does not undergo longitudinal stretch. Grifka et al first used this technique for the PDA with a diameter of $2.4-3.9 \mathrm{~mm}$, and Kumar reported that a PDA of 3-6 $\mathrm{mm}$ in patients with a body weight of less than $10 \mathrm{~kg}$ could be closed effectively and safely by this method 13 Currently, we prefer to use the 0.052 -inch coil when the minimum diameter is $\geq 2.5 \mathrm{~mm}$, as long as the aortic ampulla has sufficient size to accept loops constructed from a heavier gauze wire, because we believe that a PDA $\geq 2.5 \mathrm{~mm}$ is likely to need multiple coils for safe deployment and complete closure!,15 In this study, there was no significant difference in the rate of successful deployment and complete occlusion before and after introduction of the 0.052-inch coil. However, the number of PDA $\geq 2.5 \mathrm{~mm}$ significantly increased after introduction of a 0.052 -inch coil. Furthermore, it became easier to place the coil uneventfully without migration and procedure time was shorter, even though a PDA up to approximately $3.0 \mathrm{~mm}$ in diameter can be closed by an ordinary 0.038 -inch Gianturco coil or a Flipper coil. When we compare the outcome of PDA $\geq 4.0 \mathrm{~mm}$, the 0.052 -inch coil definitely has an advantage over coils with a smaller gauge wire. Kumar recommended using an Amplatzer duct occluder for any PDA $\geq 6 \mathrm{~mm}^{13}$ while the maximum diameter of PDA that could be closed in our study was $5.6 \mathrm{~mm}$. Consequently, the limits of our method may be around $6 \mathrm{~mm}$, even though we use multiple 0.052 -inch coils.

We deploy multiple 0.052 -inch coils simultaneously for PDA $\geq 3.5-4.0 \mathrm{~mm}$, but if they were to embolize with the stiffer coils entangled, they may be more difficult to retrieve. Consequently, we believe the Amplatzer duct occluder has definite advantages over multiple 0.052-inch coils for closing large PDAs. The role of the 0.052-inch coil with its cheaper cost will be limited to occlusion of a PDA with a diameter around $3 \mathrm{~mm}$ when the Amplatzer duct occluder is finally released in Japan.

One disadvantage of this stainless steel coil is its magnetic resonance imaging (MRI) incompatibility. As we now have a Flipper coil made from MRI compatible alloy (inconel), there may be arguments against the use of a MRI incompatible coil for PDAs that may need to be closed with multiple MRI compatible coils, even though use of such coils might increase the risk of adverse events during deployment.

Coil occlusions pre 0.052 -inch coil were in the early stages of our experience with this procedure, whereas the number of coil occlusions increased post 0.052 -inch. Our comparison between pre and post 0.052 -inch coil is neither a double-blind nor a controlled study. Consequently, some learning curve factors may have contributed to the uneventful coil deployment post 0.052 -inch coil. However, we achieved 10 consecutive uneventful coil occlusions for PDA with a minimum diameter $2.3-4.7 \mathrm{~mm}$ in the initial series post 0.052 -inch coil, despite introduction of a new device and delivery technique! ${ }^{2}$ We believe introduction of the 0.052-inch coil, rather than the learning curve, contributed to the dramatic change in outcome for medium-sized PDAs.

In conclusion, introduction of the Gianturco 0.052-inch coil decreased eventful deployment during coil occlusion for PDA $\geq 2.5 \mathrm{~mm}$, and contributed to better outcomes of coil occlusion for PDA $\geq 4 \mathrm{~mm}$. For safe deployment, a 0.052 -inch coil should be used for occlusion of PDA $\geq 2.5 \mathrm{~mm}$, and PDA up to $6 \mathrm{~mm}$ may be closed with multiple 0.052-inch coils as long as the ampulla is of sufficient size. However, surgery is indicated for PDA $\geq 6 \mathrm{~mm}$ or with a small ampulla until the Amplatzer duct occluder is approved in Japan.

\section{Acknowledgment}

We thank Dr Peter M. Olley, Professor Emeritus of Pediatrics, University of Alberta, and Dr Setsuko Olley for language consultation.

\section{References}

1. Cambier PA, Kirby WC, Wortham DC, Moore JW. Percutaneous closure of the small (less than $2.5 \mathrm{~mm}$ ) patent ductus arteriosus using coil embolization. Am J Cardiol 1992; 69: 815-816.

2. Moore JW, George L, Kirkpatrick SE, Mathewson JW, Spicer RL, Uzark K, et al. Percutaneous closure of the small patent ductus arteriosus using occluding spring coils. J Am Coll Cardiol 1994; 23: $759-765$.

3. Akagi T, Hashino K, Sugimura T, Ishii M, Eto G, Kato H. Coil occlusion of patent ductus arteriosus with detachable coils. Am Heart J 1997; 134: 538-543.

4. Tomita H, Fuse S, Akagi T, Koike K, Kamada M, Kamiya T, et al. Coil occlusion for patent ductus arteriosus in Japan. Jpn Circ J 1997; 61: $997-1003$.

5. Pass RH, Hijazi Z, Hsu DT, Lewis V, Hellenbrand WE. Multicenter USA Amplatzer patent ductus arteriosus occlusion device trial: Initial and one-year results. J Am Coll Cardiol 2004; 44: 513-519.

6. Porstmann W, Wierny L, Warnke H. Closure of persistent ductus arteriosus without thoracotomy. Ger Med Mon 1967; 12: 259-261.

7. Rashkind WJ, Mullins CE, Hellenbrand WE, Tait MA. Nonsurgical closure of patent ductus arteriosus: Clinical application of the Rashkind PDA occluder system. Circulation 1987; 75: 583-592.

8. Grifka RG. Transcatheter closure of the patent ductus arteriosus Cathet Cardiovasc Intervent 2004; 61: 554-570.

9. Grifka RG, Jones TK. Transcatheter closure of large PDA using 0.052" Gianturco coils: Controlled delivery using a bioptome catheter through a 4 French sheath. Catheter Cardiovasc Intervent 2000; 49: $301-306$.

10. Krichenko A, Benson LN, Burrows P, Moes CAF, McLaughlin P, Freedom RM. Angiographic classification of the isolated, persistently patent ductus arteriosus and implications for percutaneous catheter occlusion. Am J Cardiol 1989; 63: 877-880.

11. Sommer RJ, Gutierrez A, Lai WW, Parness IA. Use of preformed nitinol snare to improve transcatheter coil delivery in occlusion of patent ductus arteriosus. Am J Cardiol 1994; 74: 836-839.

12. Tomita H, Ono Y, Miyazaki A, Tanaka T, Kimura K, Echigo S. Transcatheter occlusion of patent ductus arteriosus using a 0.052 -inch coil: Immediate results. Circ J 2000; 64: 520-523.

13. Kumar RK, Anil SR, Kannan BRJ, Phillip A, Sivakumar K. Biotomeassisted coil occlusion of moderate-large patent ductus arteriosus in infants and small children. Cathet Cardiovasc Intervent 2004; 62: 266-271.

14. Tomita H, Fuse S, Akagi T, Matsumoto Y, Murakami Y, Shiraya H, et al. Hemolysis complicating coil occlusion of patent ductus arteriosus. Cathet Cardiovasc Diagn 1998; 43: 50-53.

15. Hijazi ZM, Lloyd TR, Beekman RH III, Geggel RL. Transcatheter closure with single or multiple coils of patent ductus arteriosus in infants weighing $\leq 8 \mathrm{~kg}$ : Retrograde versus antegrade approach. Am Heart J 1996; 132: 827-835. 